\title{
BEBERAPA SIFAT DARI SUBGRUP FUZZY
}

\author{
PUTRI EKA RIANDANI, NOVA NOLIZA BAKAR, MONIKA RIANTI HELMI \\ Program Studi Matematika, \\ Fakultas Matematika dan Ilmu Pengetahuan Alam, Universitas Andalas, \\ Kampus UNAND Limau Manis Padang, Indonesia, \\ email : putriekariandani@gmail.com
}

\begin{abstract}
Abstrak. Pada tulisan ini akan dibahas beberapa sifat dari subgrup fuzzy. Untuk itu, diperlukan konsep-konsep tentang grup, subgrup, himpunan fuzzy, dan subgrup fuzzy. Diberikan $G$ adalah grup, pemetaan $\mu: G \longrightarrow[0,1]$ disebut himpunan fuzzy dari $G$. Selanjutnya didefinisikan bahwa $\mu$ subgrup fuzzy dan dibuktikan beberapa sifat dari subgrup fuzzy tersebut seperti subgrup fuzzy normal, normalizer fuzzy, serta syarat perlu dan syarat cukup agar $\mu$ adalah subgrup fuzzy normal dari $G$.

Kata Kunci: Grup, subgrup, himpunan fuzzy, subgrup fuzzy, subgrup fuzzy normal, normalizer
\end{abstract}

\section{Pendahuluan}

Himpunan fuzzy pertama kali diperkenalkan pada tahun 1965 oleh L.A. Zadeh. Zadeh mendefinisikan suatu himpunan fuzzy $\mu$ dalam $X$ dengan fungsi keanggotaan $f_{\mu}(x)$, dimana nilai keanggotaan dari elemen-elemennya adalah bilangan riil dalam interval $[0,1][5]$. Konsep himpunan fuzzy yang terus berkembang, mendorong para peneliti untuk terus mengembangkan dan menganalisa himpunan fuzzy baik secara teoritis maupun aplikasi. Salah satunya adalah teori tentang subgrup fuzzy.

Subgrup adalah suatu subhimpunan tak kosong $H$ dari grup $G$, dimana $H$ membentuk operasi biner yang sama pada grup $G$ [1]. Misalkan didefinisikan $G$ adalah grup, suatu subhimpunan fuzzy $\mu$ dari $\mathrm{G}$ disebut subgrup fuzzy dari $G$ jika memenuhi $\mu(x y) \geq \min \{\mu(x), \mu(y)\}$ dan $\mu\left(x^{-1}\right)=\mu(x)$, untuk setiap $x, y \in G$.

Himpunan fuzzy, subgrup dan subgrup fuzzy tersebut merupakan konsep dasar pada tulisan ini, sehingga dari konsep-konsep tersebut Penulis akan membahas beberapa sifat dari subgrup fuzzy yang merupakan kajian kembali makalah [3].

\section{Subhimpunan Fuzzy}

Definisi 2.1. [2] Misalkan X adalah himpunan tak kosong. Suatu himpunan (subhimpunan) fuzzy $\mu$ dari $X$ adalah fungsi $\mu: X \longrightarrow[0,1]$.

Definisi 2.2. [2] Gabungan dari dua himpunan fuzzy $\lambda$ dan $\mu$ dari himpunan $X$ yang didefinisikan oleh $\lambda \cup \mu$ adalah subhimpunan fuzzy dari $X$, didefinisikan sebagai $(\lambda \cup \mu)(x)=\max \{\lambda(x), \mu(x)\}$, untuk setiap $x \in X$. Irisan dari dua himpunan (subhimpunan) fuzzy $\lambda$ dan $\mu$ dari $X$, ditulis $\lambda \cap \mu$, adalah subhimpunan fuzzy dari $X$ didefinisikan sebagai $(\lambda \cap \mu)(x)=\min \{\lambda(x), \mu(x)\}$, untuk setiap $x \in X$. 
58 Putri Eka Riandani dkk

\section{Beberapa Sifat dari Subgrup Fuzzy}

Pada bagian ini, akan dibahas beberapa sifat dari subgrup fuzzy.

Definisi 3.1. [2] Misalkan $G$ adalah suatu grup. Suatu subhimpunan fuzzy $\mu$ dari $G$ disebut subgrup fuzzy dari $G$ jika memenuhi:

(1) $\mu(x y) \geq \min \{\mu(x), \mu(y)\}$, untuk setiap $x, y \in G$.

(2) $\mu\left(x^{-1}\right)=\mu(x)$, untuk setiap $x \in G$.

Contoh 3.2. Diketahui bahwa $Z_{5}-\{\overline{0}\}$ adalah grup terhadap perkalian. Didefinisikan $\mu: Z_{5}-\{\overline{0}\} \longrightarrow[0,1]$, dengan:

$$
\mu(x)= \begin{cases}1 & , x=\overline{1} \\ 0,25 & , x=\overline{2}, \overline{3} \\ 0,5 & , x=\overline{4} .\end{cases}
$$

Akan ditunjukkan bahwa $\mu$ subgrup fuzzy atas grup $Z_{5}-\{\overline{0}\}$. Karena $\mu$ adalah suatu fungsi dimana $\mu(x) \in[0,1]$, untuk setiap $x \in Z_{5}-\{\overline{0}\}$, maka $\mu$ merupakan subhimpunan fuzzy atas grup $Z_{5}-\{\overline{0}\}$.

Selanjutnya akan diselidiki apakah $\mu$ merupakan subgrup fuzzy atas grup $Z_{5}-$ $\{\overline{0}\}$. Perhatikan Tabel 1 - Tabel 3.

Tabel 1. Tabel Cayley dari $Z_{5}-\{\overline{0}\}$ terhadap perkalian

\begin{tabular}{|c|c|c|c|c|}
\hline$\cdot$ & $\overline{1}$ & $\overline{2}$ & $\overline{3}$ & $\overline{4}$ \\
\hline$\overline{1}$ & $\overline{1}$ & $\overline{2}$ & $\overline{3}$ & $\overline{4}$ \\
$\overline{2}$ & $\overline{2}$ & $\overline{4}$ & $\overline{1}$ & $\overline{3}$ \\
$\overline{3}$ & $\overline{3}$ & $\overline{1}$ & $\overline{4}$ & $\overline{2}$ \\
$\overline{4}$ & $\overline{4}$ & $\overline{3}$ & $\overline{2}$ & $\overline{1}$ \\
\hline
\end{tabular}

Berdasarkan Tabel 2, diperoleh $\mu(x y) \geq \min \{\mu(x), \mu(y)\}$, untuk setiap $x, y \in$ $Z_{5}-\{\overline{0}\}$, dan dari Tabel 3 diperoleh $\mu\left(x^{-1}\right)=\mu(x)$, untuk setiap $x \in Z_{5}-\{\overline{0}\}$. Karena itu $\mu$ merupakan subgrup fuzzy atas grup $Z_{5}-\{\overline{0}\}$.

Definisi 3.3. [4] Misalkan $\mu$ subgrup fuzzy dari grup $G$. Untuk $a \in G$, koset kiri (atau kanan) fuzzy a $\mu$ (atau $\mu a$ ) dari $G$ oleh a dan $\mu$ didefinisikan sebagai $(a \mu)(x)=$ $\mu\left(a^{-1} x\right)\left(\right.$ atau $\left.(\mu a)(x)=\mu\left(x a^{-1}\right)\right)$ untuk setiap $x \in G$.

Koset kiri dan koset kanan fuzzy dikatakan sama $(a \mu=\mu a)$ jika untuk setiap $x \in G$, berlaku $(a \mu)(x)=(\mu a)(x)$.

Contoh 3.4. Diketahui bahwa $Z_{5}-\{\overline{0}\}$ adalah grup terhadap perkalian. Didefinisikan $\mu: Z_{5}-\{\overline{0}\} \longrightarrow[0,1]$, dengan:

$$
\mu(x)= \begin{cases}1 & , x=\overline{1} \\ 0,25 & , x=\overline{2}, \overline{3} \\ 0,5 & , x=\overline{4}\end{cases}
$$


Tabel 2. Operasi subhimpunan fuzzy $\mu$

\begin{tabular}{|c|c|c|c|c|c|c|}
\hline$x$ & $y$ & $x y$ & $\mu(x)$ & $\mu(y)$ & $\mu(x y)$ & $\min \{\mu(x), \mu(y)\}$ \\
\hline$\overline{1}$ & $\overline{1}$ & $\overline{1}$ & 1 & 1 & 1 & 1 \\
$\overline{1}$ & $\overline{2}$ & $\overline{2}$ & 1 & 0,25 & 0,25 & 0,25 \\
$\overline{1}$ & $\overline{3}$ & $\overline{3}$ & 1 & 0,25 & 0,25 & 0,25 \\
$\overline{1}$ & $\overline{4}$ & $\overline{4}$ & 1 & 0,5 & 0,5 & 0,5 \\
$\overline{2}$ & $\overline{1}$ & $\overline{2}$ & 0,25 & 1 & 0,25 & 0,25 \\
$\overline{2}$ & $\overline{2}$ & $\overline{4}$ & 0,25 & 0,25 & 0,5 & 0,25 \\
$\overline{2}$ & $\overline{3}$ & $\overline{1}$ & 0,25 & 0,25 & 1 & 0,25 \\
$\overline{2}$ & $\overline{4}$ & $\overline{3}$ & 0,25 & 0,5 & 0,25 & 0,25 \\
$\overline{3}$ & $\overline{1}$ & $\overline{3}$ & 0,25 & 1 & 0,25 & 0,25 \\
$\overline{3}$ & $\overline{2}$ & $\overline{1}$ & 0,25 & 0,25 & 1 & 0,25 \\
$\overline{3}$ & $\overline{3}$ & $\overline{4}$ & 0,25 & 0,25 & 0,5 & 0,25 \\
$\overline{3}$ & $\overline{4}$ & $\overline{2}$ & 0,25 & 0,5 & 0,25 & 0,25 \\
$\overline{4}$ & $\overline{1}$ & $\overline{4}$ & 0,5 & 1 & 0,5 & 0,5 \\
$\overline{4}$ & $\overline{2}$ & $\overline{3}$ & 0,5 & 0,25 & 0,25 & 0,25 \\
$\overline{4}$ & $\overline{3}$ & $\overline{2}$ & 0,5 & 0,25 & 0,25 & 0,25 \\
$\overline{4}$ & $\overline{4}$ & $\overline{1}$ & 0,5 & 0,5 & 1 & 0,5 \\
\hline
\end{tabular}

Tabel 3. Invers subhimpunan fuzzy $\mu$

\begin{tabular}{|c|c|c|c|}
\hline$x$ & $x^{-1}$ & $\mu(x)$ & $\mu\left(x^{-1}\right)$ \\
\hline$\overline{1}$ & $\overline{1}$ & 1 & 1 \\
$\overline{2}$ & $\overline{3}$ & 0,25 & 0,25 \\
$\overline{3}$ & $\overline{2}$ & 0,25 & 0,25 \\
$\overline{4}$ & $\overline{4}$ & 0,5 & 0,5 \\
\hline
\end{tabular}

Dari Contoh 3.2, diperoleh bahwa $\mu$ subgrup fuzzy. Koset kiri fuzzy a $\mu$ dihitung dan diberikan oleh:

$$
(a \mu)(x)= \begin{cases}1 & , x=\overline{2} \\ 0,25 & , x=\overline{1}, \overline{4} \\ 0,5 & , x=\overline{3}\end{cases}
$$

untuk $a=\overline{2}$.

Definisi 3.5. [2] Misalkan $\mu$ subgrup fuzzy dari grup G. Maka untuk sebarang $a, b \in$ $G$, koset tengah fuzzy a $\mu b$ dari grup $G$ didefinisikan sebagai $(a \mu b)(x)=\mu\left(a^{-1} x b^{-1}\right)$, untuk setiap $x \in G$.

Contoh 3.6. Diketahui bahwa $Z_{5}-\{\overline{0}\}$ adalah grup terhadap perkalian. Didefini- 
60 Putri Eka Riandani dkk.

sikan $\mu: Z_{5}-\{\overline{0}\} \longrightarrow[0,1]$, dengan:

$$
\mu(x)= \begin{cases}1 & , x=\overline{1} \\ 0,25 & , x=\overline{2}, \overline{3} \\ 0,5 & , x=\overline{4} .\end{cases}
$$

Dari Contoh 3.2, diperoleh bahwa $\mu$ subgrup fuzzy. Koset tengah fuzzy $a \mu b$ dihitung dan diberikan oleh:

$$
(a \mu b)(x)= \begin{cases}1 & , x=\overline{2} \\ 0,25 & , x=\overline{1}, \overline{4} \\ 0,5 & , x=\overline{3}\end{cases}
$$

untuk $a=\overline{4}$ dan $b=\overline{3}$.

Proposisi 3.7. [3] Misalkan $\mu$ subhimpunan fuzzy dari $G$, maka persamaan berikut ekivalen untuk setiap $a, x \in G$.

(1) $\mu\left(a x a^{-1}\right) \geq \mu(x)$.

(2) $\mu\left(a x a^{-1}\right)=\mu(x)$.

(3) $\mu(a x)=\mu(x a)$.

(4) $a \mu=\mu a$.

(5) $a \mu a^{-1}=\mu$.

Bukti. Misalkan $\mu$ subhimpunan fuzzy dari $G$, maka $\mu$ subgrup fuzzy dari $G$ (Definisi 3.1).

$(1 \rightarrow 2)$ Misal $\mu\left(a x a^{-1}\right) \geq \mu(x)$, untuk setiap $a, x \in G$.

Akan ditunjukkan $\mu\left(a x a^{-1}\right)=\mu(x)$.

Karena $\mu\left(a x a^{-1}\right) \geq \mu(x)$, maka jelas $\mu\left(a x a^{-1}\right)=\mu(x)$.

$(2 \rightarrow 3)$ Misal $\mu\left(a x a^{-1}\right)=\mu(x)$, untuk setiap $a, x \in G$. Akan ditunjukkan $\mu(a x)=\mu(x a)$.

Perhatikan bahwa:

$$
\begin{aligned}
\mu(a x) & =\mu(a x e) \\
& =\mu\left(a x\left(a a^{-1}\right)\right) \\
& =\mu\left(a(x a) a^{-1}\right) \\
& =\mu(x a) .
\end{aligned}
$$

Jadi $\mu(a x)=\mu(x a)$, untuk setiap $a, x \in G$.

$(3 \rightarrow 4)$ Misal $\mu(a x)=\mu(x a)$, untuk setiap $a, x \in G$. Akan ditunjukkan $a \mu=\mu a$, yaitu dengan menunjukkan $(a \mu)(x)=(\mu a)(x)$, untuk setiap $x \in G$. Perhatikan bahwa:

$$
\begin{aligned}
(a \mu)(x) & =\mu\left(a^{-1} x\right) \\
& =\mu\left(x a^{-1}\right) \\
& =(\mu a)(x) .
\end{aligned}
$$

Jadi $(a \mu)(x)=(\mu a)(x)$, untuk setiap $x \in G$. 
$(4 \rightarrow 5)$ Misal $a \mu=\mu a$. Akan ditunjukkan $a \mu a^{-1}=\mu$ yaitu dengan menunjukkan $\left(a \mu a^{-1}\right)(x)=\mu(x)$, untuk setiap $x \in G$.

Perhatikan bahwa:

$$
\begin{aligned}
\left(a \mu a^{-1}\right)(x) & =\mu\left(a^{-1} x\left(a^{-1}\right)^{-1}\right) \\
& =\mu\left(a^{-1}(x a)\right) \\
& =(a \mu)(x a) \\
& =(\mu a)(x a) \\
& =\mu\left((x a) a^{-1}\right) \\
& =\mu(x) .
\end{aligned}
$$

Jadi $\left(a \mu a^{-1}\right)(x)=\mu(x)$, untuk setiap $x \in G$.

$(5 \rightarrow 1)$ Misal $a \mu a^{-1}=\mu$. Akan ditunjukkan $\mu\left(a x a^{-1}\right) \geq \mu(x)$.

Diketahui $a \mu a^{-1}=\mu$. Andaikan $\mu\left(a x a^{-1}\right)<\mu(x)$.

Perhatikan bahwa

$$
\begin{aligned}
\mu\left(a x a^{-1}\right) & <\mu(x) \\
& =\mu(\text { exe }) \\
& =\mu\left(\left(a^{-1} a\right) x\left(a^{-1} a\right)\right) \\
& =\mu\left(a^{-1}\left(a x a^{-1}\right) a\right) \\
& =\mu\left(a^{-1}\left(a x a^{-1}\right)\left(a^{-1}\right)^{-1}\right) \\
& =a \mu a^{-1}\left(a x a^{-1}\right) .
\end{aligned}
$$

Ini berarti $\mu<a \mu a^{-1}$. Kontradiksi dengan $\mu=a \mu a^{-1}$, sehingga haruslah $\mu\left(a x a^{-1}\right) \geq \mu(x)$.

Jadi $\mu\left(a x a^{-1}\right) \geq \mu(x)$, untuk setiap $a, x \in G$.

Proposisi 3.8. [3] Jika $\mu$ subgrup fuzzy dari grup $G$, maka $g \mu g^{-1}$ juga subgrup fuzzy dari grup $G$, untuk setiap $g \in G$.

Bukti. Misal $\mu$ subgrup fuzzy dari grup $G$ dan $g \in G$. Akan ditunjukkan $g \mu g^{-1}$ juga subgrup fuzzy dari grup $G$, yaitu dengan menunjukkan:

(1) $g \mu g^{-1}(x y) \geq \min \left\{g \mu g^{-1}(x), g \mu g^{-1}(y)\right\}$, untuk setiap $x, y \in G$.

(2) $g \mu g^{-1}\left(x^{-1}\right)=g \mu g^{-1}(x)$, untuk setiap $x \in G$.

(1) Ambil $x, y \in G$, maka

$$
\begin{aligned}
g \mu g^{-1}(x y) & =\mu\left(g^{-1}(x y)\left(g^{-1}\right)^{-1}\right) \\
& =\mu\left(g^{-1}(x y) g\right) \\
& =\mu\left(g^{-1}\left(x g g^{-1} y\right) g\right) \\
& =\mu\left(\left(g^{-1} x g\right)\left(g^{-1} y g\right)\right) \\
& \geq \min \left\{\mu\left(g^{-1} x g\right), \mu\left(g^{-1} y g\right)\right\} \\
& =\min \left\{g \mu g^{-1}(x), g \mu g^{-1}(y)\right\} .
\end{aligned}
$$


(2) Ambil $x \in G$, maka

$$
\begin{aligned}
g \mu g^{-1}\left(x^{-1}\right) & =\mu\left(g^{-1} x^{-1}\left(g^{-1}\right)^{-1}\right) \\
& =\mu\left(g^{-1} x^{-1} g\right) \\
& =\mu\left(\left(g^{-1} x g\right)^{-1}\right) \\
& =\mu\left(g^{-1} x g\right) \\
& =g \mu g^{-1}(x) .
\end{aligned}
$$

Dari (1) dan (2), diperoleh bahwa $g \mu g^{-1}$ juga subgrup fuzzy dari grup $G$.

Definisi 3.9. [2] Misalkan $G$ grup, subgrup fuzzy $\mu$ dari $G$ disebut normal jika $\mu(x)=\mu\left(y^{-1} x y\right)$, untuk setiap $x, y \in G$.

Proposisi 3.10. [3] Irisan sebarang dua subgrup fuzzy normal dari grup G juga subgrup fuzzy normal dari grup $G$.

Bukti. Misal $\lambda$ dan $\mu$ dua subgrup fuzzy normal dari grup $G$. Akan ditunjukkan $\lambda \cap \mu$ subgrup fuzzy normal dari $G$, yaitu dengan menunjukkan:

(1) $\lambda \cap \mu$ subgrup fuzzy.

(2) $(\lambda \cap \mu)(x)=(\lambda \cap \mu)\left(y^{-1} x y\right)$, untuk setiap $x, y \in G$.

(1) Akan ditunjukkan $\lambda \cap \mu$ subgrup fuzzy.

(i) Ambil $x, y \in G$, maka

$$
\begin{aligned}
(\lambda \cap \mu)(x y) & =\min \{\lambda(x y), \mu(x y)\} \\
& \geq \min \{\min \{\lambda(x), \lambda(y)\}, \min \{\mu(x), \mu(y)\}\} \\
& =\min \{\min \{\lambda(x), \mu(x)\}, \min \{\lambda(y), \mu(y)\}\} \\
& =\min \{(\lambda \cap \mu)(x),(\lambda \cap \mu)(y)\} .
\end{aligned}
$$

(ii) Ambil $x \in G$, maka

$$
\begin{aligned}
(\lambda \cap \mu)\left(x^{-1}\right) & =\min \left\{\lambda\left(x^{-1}\right), \mu\left(x^{-1}\right)\right\} \\
& =\min \{\lambda(x), \mu(x)\} \\
& =(\lambda \cap \mu)(x) .
\end{aligned}
$$

Dari (i) dan (ii), diperoleh bahwa $\lambda \cap \mu$ subgrup fuzzy.

(2) Akan ditunjukkan subgrup fuzzy $\lambda \cap \mu$ adalah normal. Ambil $x \in G$, maka

$$
\begin{aligned}
(\lambda \cap \mu)(x) & =\min \{\lambda(x), \mu(x)\} \\
& =\min \left\{\lambda\left(y^{-1} x y\right), \mu\left(y^{-1} x y\right)\right\} \\
& =(\lambda \cap \mu)\left(y^{-1} x y\right) .
\end{aligned}
$$

Dari (1) dan (2), diperoleh bahwa $\lambda \cap \mu$ subgrup fuzzy normal.

Definisi 3.11. [6] Misalkan $G$ suatu grup dan $\mu$ subgrup fuzzy dari G. Misalkan $N(\mu)=\left\{a \in G \mid \mu\left(a x a^{-1}\right)=\mu(x)\right.$, untuk setiap $\left.x \in G\right\}$, maka $N(\mu)$ disebut normalizer fuzzy dari $\mu$. 
Proposisi 3.12. [3] Normalizer fuzzy dari $\mu$ dengan $\mu$ subgrup fuzzy dari $G$ adalah subgrup dari $G$.

Bukti. Misal $\mu$ subgrup fuzzy dari grup G. $N(\mu)=\left\{a \in G \mid \mu\left(a x a^{-1}\right)=\mu(x)\right.$, untuk setiap $x \in G\}$ adalah normalizer dari $\mu$. Akan ditunjukkan $N(\mu)$ subgrup dari $G$ yaitu dengan menunjukkan:

(1) $N(\mu) \subset G$ dan $N(\mu) \neq \emptyset$.

(2) Untuk setiap $m, n \in N(\mu)$, berlaku $m n \in N(\mu)$.

(3) Untuk setiap $m \in N(\mu)$, berlaku $m^{-1} \in N(\mu)$.

(1) Berdasarkan definisi $N(\mu)$ jelas bahwa $N(\mu) \subset G$.

Selanjutnya, karena $G$ grup, maka terdapat $e \in G$ sehingga

$$
\begin{aligned}
\mu\left(e x e^{-1}\right) & =\mu\left((e x) e^{-1}\right) \\
& =\mu(x) .
\end{aligned}
$$

Karena $e \in G$ dan $\mu\left(e x e^{-1}\right)=\mu(x)$, untuk setiap $x \in G$, maka $e \in N(\mu)$, sehingga $N(\mu) \neq \emptyset$.

(2) Misal $m, n \in N(\mu)$.

Karena $m \in N(\mu)$, maka $m \in G$ dan $\mu\left(m x m^{-1}\right)=\mu(x)$, untuk setiap $x \in G$. Karena $n \in N(\mu)$, maka $n \in G$ dan $\mu\left(n x n^{-1}\right)=\mu(x)$, untuk setiap $x \in G$.

Akan ditunjukkan $m n \in N(\mu)$, yaitu $m n \in G$ dan $\mu\left((m n) x(m n)^{-1}\right)=\mu(x)$. Perhatikan bahwa:

$$
\begin{aligned}
\mu\left((m n) x(m n)^{-1}\right) & =\mu\left((m n) x\left(n^{-1} m^{-1}\right)\right. \\
& =\mu\left((m n)\left(x n^{-1} m^{-1}\right)\right) \\
& =\mu\left(\left(x n^{-1} m^{-1}\right)(m n)\right) \\
& =\mu\left(x n^{-1}\left(m^{-1} m\right) n\right) \\
& =\mu\left(x\left(n^{-1} n\right)\right) \\
& =\mu(x) .
\end{aligned}
$$

Ini berarti $m n \in N(\mu)$.

(3) Misal $m \in N(\mu)$.

Karena $m \in N(\mu)$, maka $m \in G$ dan $\mu\left(m x m^{-1}\right)=\mu(x)$, untuk setiap $x \in G$. Akan ditunjukkan $m^{-1} \in N(\mu)$, yaitu $m^{-1} \in G$ dan $\mu\left(m^{-1} x\left((m)^{-1}\right)^{-1}\right)=\mu(x)$. Perhatikan bahwa:

$$
\begin{aligned}
\mu\left(m^{-1} x\left((m)^{-1}\right)^{-1}\right) & =\mu\left(m^{-1} x m\right) \\
& =\mu\left(\left(m^{-1} x\right) m\right) \\
& =\mu\left(m\left(m^{-1} x\right)\right) \\
& =\mu\left(\left(m m^{-1}\right) x\right) \\
& =\mu(x) .
\end{aligned}
$$

Ini berarti $m^{-1} \in N(\mu)$.

Dari (1), (2), dan (3), diperoleh bahwa $N(\mu)$ subgrup dari $G$. 
64 Putri Eka Riandani dkk.

Akibat 3.13. $\mu$ subgrup fuzzy normal dari $G$ jika dan hanya jika $N(\mu)=G$.

\section{Bukti.}

$(\Rightarrow)$ Misal $\mu$ subgrup fuzzy normal dari G. Akan ditunjukkan $N(\mu)=G$, yaitu dengan menunjukkan:

(1) $N(\mu) \subset G$.

(2) $G \subset N(\mu)$.

(1) Berdasarkan definisi $N(\mu)$ jelas bahwa $N(\mu) \subset G$.

(2) Misal $z \in G$. Akan ditunjukkan $z \in N(\mu)$.

Karena $z \in G$ dan $\mu$ subgrup fuzzy normal dari G, maka $\mu(x)=\mu\left(z^{-1} x z\right)$, untuk setiap $x, z \in G$. Berarti untuk $z \in G$ berlaku $\mu(x)=\mu\left(z^{-1} x z\right)$, untuk setiap $x \in G$. Dengan perkataan lain $z \in N(\mu)$.

Oleh karena itu $G \subset N(\mu)$

Dari (1) dan (2) diperoleh bahwa $N(\mu)=G$.

$(\Leftarrow)$ Misal $N(\mu)=G$. Akan ditunjukkan $\mu$ subgrup fuzzy normal dari $G$.

Karena $N(\mu)=\left\{a \in G \mid \mu\left(a x a^{-1}\right)=\mu(x)\right.$, untuk setiap $\left.x \in G\right\}$, maka jelas bahwa $\mu\left(\right.$ axa $\left.^{-1}\right)=\mu(x)$, untuk $a \in G$ dan $x \in G$. Ini berarti $\mu$ adalah subgrup fuzzy normal.

\section{Ucapan Terima kasih}

Penulis mengucapkan terima kasih kepada Bapak Admi Nazra, Bapak I Made Arnawa, dan Ibu Susila Bahri yang telah memberikan masukan dan saran sehingga paper ini dapat diselesaikan dengan baik.

\section{Daftar Pustaka}

[1] Herstein, I. N. 1975. Topics in Algebra,2nd edition. New York : John Wiley dan Sons.

[2] Kandasamy, W. B. V. 2003. Smarandache Fuzzy Algebra. USA : American Research Press Rehoboth.

[3] Mashour, A. S., M.H. Ghanim dan F.I. Sidky. 1990. Normal fuzzy subgroups. Univ. U Novom Sadu Zb.Rad.Prirod.-Mat.Fak.Ser.Mat. 20(2): 53 - 59.

[4] Onasanya, B.O., dan S.A. Ilori. 2014. On Cosets and Normal Subgroups. International J. Math. Combin. 3: $35-40$.

[5] Yunjie Z. dan Dong Yu. Tanpa tahun. On Fuzzy Abelian Subgroup.

[6] Zadeh, L.A. 1965. Fuzzy Sets. Information and Control. 8: 338 - 353. 